\title{
RATIONAL APPROXIMATION AND SWISS CHEESES OF POSITIVE AREA
}

\author{
By Leon Brown and LeE Rubel
}

\begin{abstract}
Let $J$ and $K$ be two compact sets in the complex plane such that $K \backslash J$ has zero planar measure. If $R(J)=C(J)$ then $R(K)=C(K)$. This result is used to produce many Swiss cheeses $K$ of positive area, for which $R(K)=C(K)$.
\end{abstract}

For any compact set $K$ in the complex plane, let $C(K)$ and $R(K)$ denote, respectively, the algebra of continuous functions on $K$, and the subalgebra of functions which are uniformly approximable on $K$ by rational functions with poles off $K$. Hartogs and Rosenthal proved in [2] that if $m_{2}(K)=0$ (where $m_{2}$ denotes planar Lebesgue measure), then $R(K)=C(K)$. We extend this theorem here, and apply it to get new examples of Swiss cheeses $K$ with $R(K)=C(K)$, yet $m_{2}(K)>0$.

THEOREM. Let $J$ and $K$ be compact sets such that $m_{2}(K \backslash J)=0$. If $R(J)=$ $C(J)$ then $R(K)=C(K)$.

The proof of this result depends on the following. Let $\mu$ be a finite measure with compact support in the complex plane. The Cauchy transform of $\mu$ is defined by $\mu^{\wedge}(w)=\int(z-w)^{-1} d \mu(z)$. It is the convolution of $\mu$ with the locally integrable function $1 / z$. So the integral defining $\mu^{\wedge}$ converges absolutely except for $w$ belonging to a set of zero planar measure. Clearly, $\mu^{\wedge}$ is analytic off the closed support of $\mu$. A converse of this statement is true.

Proposition 1. (See [1], Theorem 8.2.) Let $\mu$ be a finte measure of compact support in the plane. Suppose $U$ is an open set, and $f$ is a function analytic on $U$ such that $f=\mu^{\wedge}$ almost everywhere with respect to $m_{2}$ on $U$. Then $|\mu|(U)=0$.

Proof of Theorem 1. We show that any measure $\mu$ with support in $K$ which is orthogonal to $R(K)$ must be the zero measure. In Proposition 1 , set $f \equiv 0$ and $U=C J$. Since $\mu \perp R(K), \mu^{\wedge}=0$ on $C K$. Since $m_{2}(K \backslash J)=0$, we have $\mu^{\wedge}=f$ almost

1980 Mathematics Subject Classification Numbers 41A20, 30E10.

This research was partially supported by the National Science Foundation.

Received October 27, 1980. 
everywhere with respect to $m_{2}$ on $U$ because $U=C J=(K \backslash J) \cup(C J \cap C K)$. Thus the support of $\mu$ is contained in $J$. Also, $\mu^{\wedge}=0$ throughout $C J$ because $C K \cap C J$ is a dense open subset of $C J$. So $\mu \perp R(J)$, and thus $\mu=0$ because $R(J)=C(J)$, and the theorem is proved.

We shall consider some special cases of the theorem. If we take $J$ to be a singleton, then we get the theorem of Hartogs-Rosenthal by a different proof from theirs. In another direction, we will construct a Swiss cheese $K$ with $m_{2}(K)>0$, yet $R(K)=C(K)$. Such an example, based on different ideas, was given by C.R. Putnam in [3]. Let us be more detailed.

If $D_{n}, n=1,2,3, \cdots$ are open discs contained in the unit disc $D$, with the $D_{n}$ having disjoint closures, and with $\cup D_{n}$ dense in $D$, then $K=D \backslash \cup D_{n}$ is called a "Swiss cheese." Certain Swiss cheeses provide the simplest examples of compact sets $K$ with empty interior for which $R(K) \neq C(K)$. These are the ones for which $\Sigma r_{n}<\infty$, where $r_{n}$ is the radius of $D_{n}$. The Hartogs-Rosenthal theorem implies on the other hand that if $m_{2}(K)=0$, then $R(K)=C(K)$. Putnam in [3] extended this result to show that if $K$ is a compact set which is "areally disconnected," then $R(K)=C(K)$. A corollary to this result if that is there exists a set of real numbers $\{t\}$ dense on the real line for which each of the vertical lines $\operatorname{Re}(z)=t$ intersects $K$ on a set of zero linear measure, then $R(K)$ $=C(K)$. Using this corollary he constructs a Swiss cheese so that $m_{2}(K)>0$ and $R(K)=C(K)$.

Here is how we can use our Theorem to produce many other such examples. Let $J$ be any compact subset of $D$ such that $m_{2}(J)>0$ and $R(J)=C(J)$. For example, $J$ could be an arc of positive area or a Cantor set of positive area, in which cases Mergelyan's Theorem (see [1], Theorem 9.1) shows that $P(J)=$ $C(J)$ so that $R(J)=C(J)$. (Here $P(J)$ is the class of functions uniformly approximable on $J$ by polynomials.) Now just construct the Swiss cheese $K$ so that $K \supseteqq J$ and $m_{2}(K \backslash J)=0$. This can easily be achieved by proper choice of the $D_{n}$. Clearly, $m_{2}(K)>0$, and yet our Theorem implies that $R(K)=C(K)$.

Remark. By a slight variation of the above proof, one can prove the following. Let $A(K)$ be the algebra of continuous functions on $K$ that are analytic in the interior of $K$. Suppose now that $J$ and $K$ are compact sets with $m_{2}(K \backslash J)$ $=0$ and so that $J \backslash K$ has empty interior. If $A(J)=R(J)$, then it follows that $A(K)=R(K)$.

\section{REFERENCES}

[1] T.W. Gamelin, Uniform Algebras, Prentice Hall, Inc., Englewood Cliffs, New Jersey, 1969.

[2] F. Hartogs and A. Rosenthal, Über Folgen analytischer Funktionen, Math. Ann. 104 (1931), 606-610.

[3] C.R. Putnam, Rational approximation and Swiss cheeses, Michigan Math. J. 24 (1977), 193-196.

WAyne STATE University

UNIVERSITY OF ILLINOIS 